\title{
Checkpointhemmer beim Melanom: Nebenwirkungen meist geringgradig
}

\begin{abstract}
Wie stark fallen Nebenwirkungen im Zusammenhang mit der Immuntherapie bei Melanompatienten aus und wie lassen sie sich beherrschen? Antworten auf diese Fragen lassen sich an der gepoolten Auswertung von vier klinischen Studien ablesen.
\end{abstract}

$\mathrm{n}$ einer retrospektiven Studie wurden Nebenwirkungen aus vier Studien zur Nivolumab-Monotherapie näher untersucht. Die Befunde von 576 Patienten mit einem medianen Follow-up von 7,2 Monaten ließen sich verwerten. Der Fokus lag auf Nebenwirkungen mit potenziell immunologischem Auslöser, die eine regelmäßige Überwachung erforderlich machten und bei denen eine Immunsuppression mit oder ohne Hormonbehandlung nötig war.

Bei $71 \%$ wurden Nebenwirkungen im Zusammenhang mit der Immuntherapie beschrieben, bei $10 \%$ erreichten diese Grad 3 oder 4. Am häufigsten waren $\mathrm{Fa}$ tigue (24,8\%), Pruritus (17,2\%), Diarrhö $(12,7 \%)$ sowie Exantheme (12,7\%) und Übelkeit (12\%). Nebenwirkungen aller
Schweregrade mit potenziell immunologischem Auslöser wurden bei $49 \%$ der Patienten dokumentiert, solche vom Grad 3 und 4 nur bei 3,6\%. Vorrangig betroffen war die Haut mit einem Anteil von $34 \%$, gefolgt von Gastrointestinaltrakt $(13,4 \%)$, Hormonsystem (7,8\%) und Leber $(4,2 \%)$. Knapp $4 \%$ waren in der Gruppe mit möglichem immunologischen Auslöser vom Grad 3 und 4.

Um die Nebenwirkungen in den Griff zu bekommen, wurde fast jeder vierte Melanompatient mit Kortikosteroiden behandelt. Bei $16 \%$ der Patienten wurden topische Kortikosteroide gegen kutane Nebenwirkungen appliziert.

Im Allgemeinen hatten sich die immunologisch bedingten Nebenwirkungen innerhalb von einem bis zwei Mona- ten nach Therapiebeginn zurückgebildet. Mit im median fünf Wochen verlief die Rückbildung bei Veränderungen der Haut am schnellsten. Wichtig sei, frühzeitig auf die Nebenwirkungen zu reagieren.

Die objektive Ansprechrate auf die Behandlung mit dem Checkpointhemmer betrug $31,4 \%(n=181)$. Sie lag bei jenen Patienten signifikant höher, bei denen immunologisch bedingte Nebenwirkungen aufgetreten waren. Bei Patienten mit Grad 1 oder $2(\mathrm{n}=242)$ lag die Rate bei $46,7 \%$, bei Patienten mit mindestens Grad $3(n=13)$ bei 84,6\%. Ein Zusammenhang mit dem medianen progressionsfreien Überleben wurde dagegen nicht beobachtet.

Fazit: Die meisten Nebenwirkungen, die bei Melanompatienten während einer Nivolumab-Therapie auftreten, sind schwach ausgeprägt und bei entsprechendem Management nur vorübergehend.

Peter Leiner

Weber JS et al. Safety Profile of Nivolumab Monotherapy: A Pooled Analysis of Patients With Advanced Melanoma. J Clin Oncol 2017; 35: 785-92

\section{Kombinationstherapie beim mukosalen Melanom}

\author{
In einer gepoolten Datenanalyse wurde erstmals Sicherheit und Wirksamkeit \\ von Nivolumab als Monosubstanz und in Kombination mit Ipilimumab beim \\ seltenen, hochaggressiven mukosalen Melanom untersucht.
}

$F_{n}^{\mathrm{u}}$ ür die Behandlung des kutanen Melanoms stehen seit 2011 mehrere neue Agenzien zur Verfügung, darunter die Kombination Nivolumab/Ipilimumab. Es existieren jedoch sehr wenige veröffentlichte Studienergebnisse zur Wirksamkeit und Sicherheit dieser neuen Therapeutika bei anderen Melanom-Subtypen wie mukosalen Melanomen. Diese hochaggressive Subtypen entziehen sich weitgehend den traditionellen Therapieansätzen. Auch gestaltet sich die komplette chirurgische Resektion aufgrund der anatomischen Lokalisation der Tumoren schwierig. Um das Wissen zum möglichen Benefit einer Anti-PD-1-Therapie des mukosalen Melanoms zu mehren, wurde eine gepoolte Datenanalyse zu Patienten mit diesem Subtyp durchgeführt, die im Rahmen klinischer Untersuchungen entweder Nivolumab als Monoagens oder in Kombination mit Ipilimumab erhalten hatten.

Für die Analyse wurden die Daten von 889 Patienten zusammengefasst, die ausschließlich den PD-1-Rezeptorblocker erhalten hatten, davon $10 \%$ mit einem mukosalen und $75 \%$ mit einem kutanen Melanom. Zudem wurden die Daten von 35 Patienten mit einem mukosalen und 326 mit einem kutanen Melanom gepoolt, die mit der Kombination Nivolumab/Ipilimumab behandelt worden waren. Für die Gruppe unter Monotherapie und mit dem mukosalen Subtyp ermittelten die Autoren ein medianes
PFS von drei Monaten und ORR von 23,3\%. Die Zahlen für Patienten mit einem mukosalen Melanom: 23,3 Monate und 40,9\%. Vorteile in diesen beiden $\mathrm{Pa}$ rametern bescherte die Kombinationstherapie: 5,9 Monate und 11,7 Monate medianes PFS (mukosales vs. kutanes Melanom) und entsprechend ORR von $37,1 \%$ oder $60,4 \%$. Die Rate an Therapieassoziierten unerwünschten Ereignissen vom Grad 3 oder 4 war in der Kombinationsgruppe erhöht.

Fazit: Nach den Ergebnissen dieser umfangreichsten Datenanalyse zum Einsatz einer gegen PD-1 gerichteten Therapie scheint die Kombination Nivolumimab/Ipilimumab der Monotherapie mit einem dieser Substanzen überlegen zu sein bei vergleichbarem Sicherheitsprofil.

Wolfgang Zimmermann

D'Angelo SP et al. Efficacy and safety of nivolumab alone or in combination with ipilimumab in patients with mucosal melanoma: a pooled analysis. J Clin Oncol 2017; 35: 226-35 